\title{
Initial singlet and triplet spin state contributions to $\vec{p} \vec{p} \rightarrow p p \pi^{0}$
}

P. Thörngren Engblom ${ }^{a}$ 円, H.O. Meyer ${ }^{a}$, J.T. Balewski $^{a}$, W.W. Daehnick ${ }^{b}$, J. Doskow $^{a}$, W. Haeberli ${ }^{c}$, B. Lorentz ${ }^{c}$, P.V. Pancella ${ }^{d}$, R.E. Pollock ${ }^{a}$, B. von Przewoski ${ }^{a}$, F. Rathmann ${ }^{e}$, T. Rinckel ${ }^{a}$, Swapan K. Saha ${ }^{b}$, B. Schwartz ${ }^{c}$, A. Wellinghausen $^{a}$, T. Wise ${ }^{c}$

${ }^{a}$ Indiana University Cyclotron Facility, Indiana University, Bloomington, IN 47405, U.S.A. ${ }^{b}$ Department of Physics, University of Pittsburgh, Pittsburgh, PA 15260, U.S.A. ${ }^{c}$ Department of Physics, University of Wisconsin-Madison, Madison, WI 53706, U.S.A. ${ }^{d}$ Department of Physics, Western Michigan University, Kalamazoo, MI 49008, U.S.A. ${ }^{e}$ Friedrich-Alexander Universität, Erwin-Rommel Str. 1, 91508 Erlangen, Germany.

The PINTEX facility at the IUCF Cooler ring, dedicated to the study of spin dependence in nucleon-nucleon interactions, has been used to measure polarization observables of the reaction $\vec{p} \vec{p} \rightarrow p p \pi^{0}$ at beam energies between 325 and $400 \mathrm{MeV}$. The stored, polarized proton beam had spin projections both in the longitudinal and the transverse directions with respect to the beam momentum. We report here on the measurements of the relative transverse and longitudinal spin-dependent cross sections $s^{3}, \Delta \sigma_{T} / \sigma_{\text {tot }}$ and $\Delta \sigma_{L} / \sigma_{\text {tot }}$, and how from these observables the initial spin singlet and triplet cross sections are obtained. Considering angular momentum states less than or equal to one, the contribution of the Ps partial waves to the cross section can be extracted.

\section{INTRODUCTION}

For reactions with two incoming spin $1 / 2$ particles one can define the initial state cross sections ${ }^{2 S+1} \sigma_{m_{s}}$ where $S$ denotes the combined spin of the two particles and $m_{s}$ its projection. The three possible initial spin state contributions ${ }^{1} \sigma_{0},{ }^{3} \sigma_{0}$ and ${ }^{3} \sigma_{1}$ are related to $\Delta \sigma_{T}$ and $\Delta \sigma_{L}$ by isospin, parity and angular momentum conservation

$\frac{{ }^{1} \sigma_{0}}{\sigma_{t o t}}=1+\frac{\Delta \sigma_{T}}{\sigma_{t o t}}+\frac{1}{2} \frac{\Delta \sigma_{L}}{\sigma_{t o t}}$

$\frac{{ }^{3} \sigma_{0}}{\sigma_{t o t}}=1-\frac{\Delta \sigma_{T}}{\sigma_{t o t}}+\frac{1}{2} \frac{\Delta \sigma_{L}}{\sigma_{t o t}}$

$\frac{{ }^{3} \sigma_{1}}{\sigma_{t o t}}=1-\frac{1}{2} \frac{\Delta \sigma_{L}}{\sigma_{t o t}}$

${ }^{1}$ Present address:Department of Radiation Sciences, Box 535, S-75121 Uppsala, Sweden. Email: pia.thorngren@tsl.uu.se

${ }^{2}$ http://www.iucf.indiana.edu/ pintex/

${ }^{3}$ Defined as $\Delta \sigma_{T(L)}=[\sigma(\uparrow \downarrow)+\sigma(\downarrow \uparrow)]-[\sigma(\uparrow \uparrow+\sigma(\downarrow \downarrow)]$ where the arrows denote parallel and antiparallel beam/target spin combinations, either transversely $(\mathrm{T})$ or longitudinally (L) polarized. 
Near threshold only a limited number of partial waves are important and considering the allowed transitions to final states fith $L_{N N} \leq 1$ and $l_{\pi} \leq 1$, i.e. assuming $\sigma_{\text {tot }} \equiv$ $\sigma_{S s}+\sigma_{P s}+\sigma_{P p}$, we obtain the following relations

${ }^{1} \sigma_{0}=4 \sigma_{P s}$

${ }^{3} \sigma_{0}=4\left(\sigma_{S s}+\hat{\sigma}_{P p}\right)$

${ }^{3} \sigma_{1}=2\left(\sigma_{P p}-\hat{\sigma}_{P p}\right)$

where $\hat{\sigma}_{P p}$ corresponds to $S=1, m_{s}=0$ initial states that contribute to $P p$ final states but not to $\sigma_{\text {tot }}$. To which extent the Ps partial waves contribute to $\sigma_{t o t}$ can then be obtained in a model-free way [1] using equ. 1-6

$\frac{\sigma_{P s}}{\sigma_{t o t}}=\frac{1}{4}\left(1+\frac{\Delta \sigma_{T}}{\sigma_{t o t}}+\frac{1}{2} \frac{\Delta \sigma_{L}}{\sigma_{t o t}}\right)$

Theoretical models for pion production near threshold can thus be tested in an unprecedented manner using measurements of spin observables of the reaction $\vec{p} \vec{p} \rightarrow p p \pi^{0}[2]$. The results for the spin-correlation coefficient combinations $A_{\Delta}=A_{x x}-A_{y y}$, and $A_{\Sigma}=$ $A_{x x}+A_{y y}$ (the latter equivalent to $-\Delta \sigma_{T} / \sigma_{t o t}$ ) and the analyzing power, $A_{y}$, which only needed transverse beam and target polarization, were published recently [3] and the publication of the the data from the experiment using also longitudinal polarization components is in progress [1]. The simultaneous measurements of spin correlation coefficients in $\vec{p} \vec{p} \rightarrow p n \pi^{+}$will further enhance the usefulness of this experiment for resolving the theoretical issues of $N N \rightarrow N N \pi$ reactions 4 .

Section 2 contains a brief description of the experimental method which is described in detail elsewhere [5,6]. In section 3 we discuss the preliminary results of the measurements using polarized beam with longitudinal components, and the possible implications.

\section{THE EXPERIMENT}

The vertically polarized beam was injected into the IUCF Cooler ring at $197 \mathrm{MeV}$ and then accelerated to $325,350,375$ or $400 \mathrm{MeV}$. The longitudinal component of the beam polarization was achieved using one superconducting solenoid and the three existing solenoids in the cooling section operated in a non-compensating mode. At the injection energy the polarization at the target was almost entirely longitudinal but since the solenoid fields remained constant, after acceleration only partially longitudinal beam polarization could be achieved. The major components were along and vertical to the beam momentum and one minor component was sideways. The beam spin direction was reversed at each new injection.

The target was polarized atomic hydrogen in a gas storage cell fed by an atomic beam source [7]. The target thickness was of the order of $10^{13}$ atoms $/ \mathrm{cm}^{2}$. The spin direction was changed every two seconds, along the $\pm x, \pm y$ and $\pm z$ axes, by switching weak guide fields $(\sim 0.3-0.6 \mathrm{mT})$, supplied by Helmholtz-like coils external to the target chamber. A total of 12 beam/target spin combinations were used. The four-momenta of the two

${ }^{4} L_{N N}$ is the angular momentum between the two nucleons and $l_{\pi}$ is the angular momentum of the pion with respect to the nucleon pair.

${ }^{5} \mathrm{Sp}$ is forbidden by parity and angular momentum conservation 


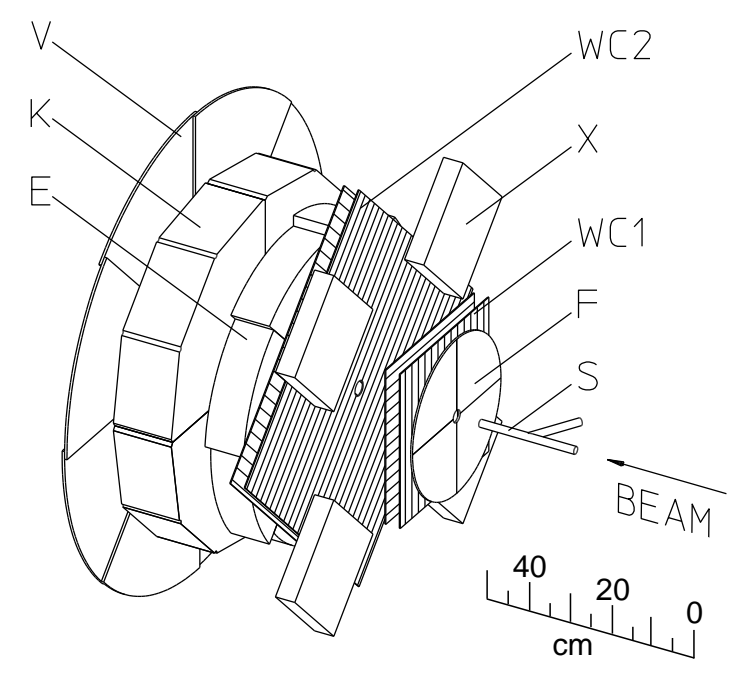

Figure 1. The forward detector set-up with an angular coverage of $5^{\circ} \leq \theta_{l a b} \leq 32^{\circ}$. The different parts are indicated: S: storage cell, F: front detector used for time-of-flight measurements, E, K: stopping scintillators with eight and four segments, thicknesses 10 and $15 \mathrm{~cm}$ respectively, V: veto detector, $\mathrm{WC1} 1,2$ : wire chambers, $\mathrm{X}$ : four monitor scintillators for measuring $p p$ elastic scattering at $\theta_{l a b}=45^{\circ}$ and $\phi= \pm 45^{\circ}$ and $\pm 135^{\circ}$.

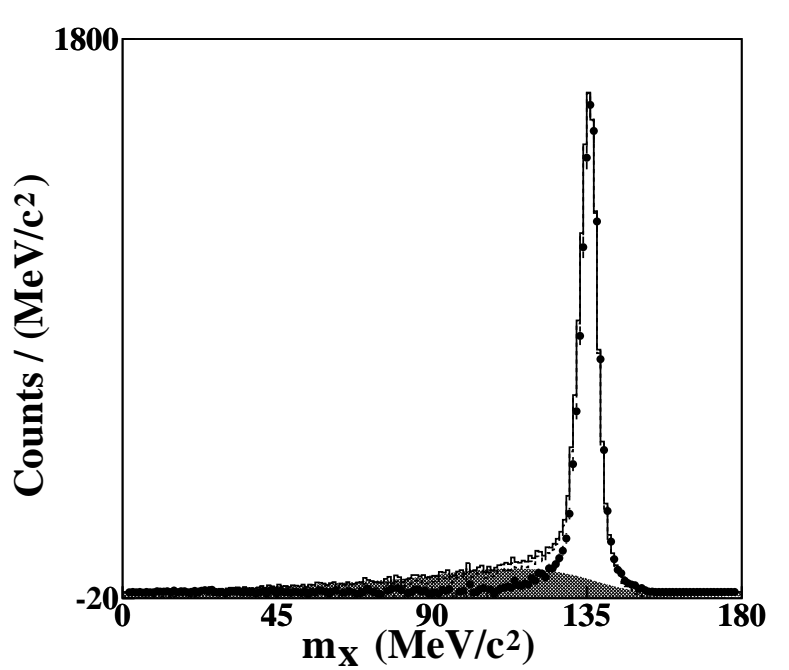

Figure 2. The unpolarized distribution of the missing mass at $325 \mathrm{MeV}$ beam energy in bins of $1 \mathrm{MeV} / \mathrm{c}^{2}$. The shaded area indicates the background which has been normalized to the area corresponding to invariant masses below $100 \mathrm{MeV} / \mathrm{c}^{2}$. The filled circles represent the data after the background has been subtracted, the statistical errors are shown. The resolution (FWHM) of the background subtracted peak is 7.2 $\mathrm{MeV} / \mathrm{c}^{2}$.

outgoing protons were measured in a cylindrically symmetric forward detector stack, see Fig. [1, which allowed for the missing mass, $m_{x}$, of the third particle to be calculated. In Fig. 2 the $m_{x}$ distribution is shown for the data set with longitudinal and transverse beam spin components summed over all spin states at a beam energy of $325 \mathrm{MeV}$. The shape of the background in the spectrum was well reproduced by data taken with nitrogen gas instead of hydrogen in the target.

Full coverage of phase space was achieved with the exception of the loss of events when protons go undetected into the beampipe, i.e. $30-22 \%$ of phase space distributed events were lost at 325-400 MeV. In an investigation of systematic errors the spin observables were studied as a function of an artificial $\theta_{l a b}$ cut-off using an effective-range expansion for the $p p$ final state interaction [8]. The corrections applied for the relative spin-dependent cross sections were less than the statistical errors.

The cross-ratio method [9] which eliminates asymmetries due to detector acceptance and spin-dependent luminosities, and division by the product of the beam and target

${ }^{6} \Delta \sigma_{T(L)} / \sigma_{\text {tot }}=\frac{2}{P Q} \frac{\sqrt{R}-1}{\sqrt{R}+1}$ where $R$ is the ratio of the yields, $Y_{\uparrow \downarrow} Y_{\downarrow \uparrow} / Y_{\uparrow \uparrow} Y_{\downarrow \downarrow}$ and $P Q$ is the product of the beam and target polarization. 
polarization projections, $P_{y} Q_{y}$ or $P_{z} Q_{z}$, give $\Delta \sigma_{T} / \sigma_{\text {tot }}$ and $\Delta \sigma_{L} / \sigma_{\text {tot }}$ from the spinsorted and backgroundsubtracted yields of the $\pi^{0}$. The values of $P_{y} Q_{y}$ and $P_{z} Q_{z}$ are obtained. by detecting $p p$ elastic scattering at $\theta_{l a b}=45^{\circ}$ simultaneously with the pion production and measuring the $p p$ spin correlation coefficients $A_{\Delta}$ and $A_{z z}$ which are large and known with high precision at this angle 10 13].

\section{PRELIMINARY RESULTS AND DISCUSSION}

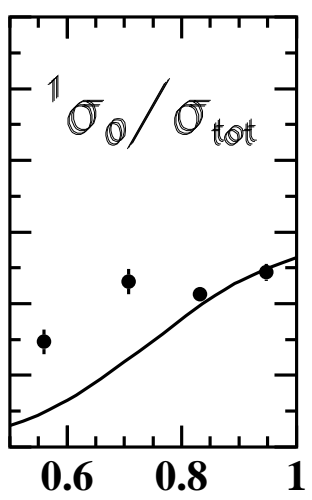

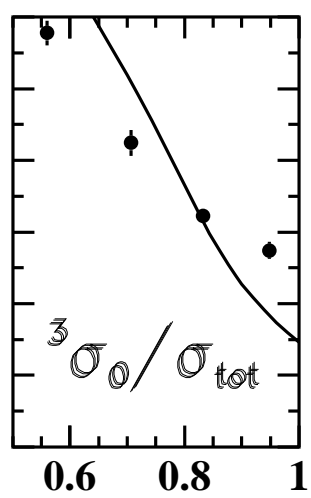

$\eta$

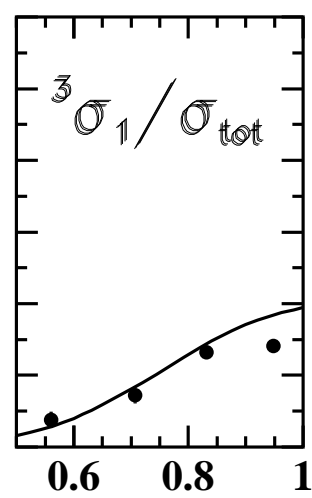

Figure 3. The data points are the preliminary results for the initial spin state cross sections. The curves are the predictions by the Jülich meson-exchange model (see text). The divisions on the y-axis are arbitrary.

The analysis of the experiment described here is near completion [1]. Preliminary results using equ. 1-3 are shown in Fig. 3. Also depicted are curves representing predictions by a microscopic model calculation, which is based on direct production and off-shell rescattering diagrams. Contributions from heavy meson-exchange $(\omega)$ are added and the $\Delta$-isobar is included explicitly [14,15]. Previous comparison with data yielded qualitative agreement for $-A_{\Sigma}$ and $A_{y}$, but disagreed completely with the data on $A_{\Delta}$ [5]. Fig. 3 indicates that ${ }^{3} \sigma_{1}$, the initial state cross section contributing only to $\mathrm{Pp}$ final states, contains the amplitudes quantitavely best reproduced by theory so far.

In [1] it is shown that the energy dependence assumed for the Ps and Pp partial waves, i.e. the proportionality to $\eta^{6}$ and $\eta^{8}$ respectively, is valid for the measured energy range. The magnitude of the Ps cross section is calculated using equ. 7 , it increases from about $18 \%$ to $30 \%$ for bombarding energies in the range from 325 to $400 \mathrm{MeV}$. At $310 \mathrm{MeV}$ there are recent reports of a significant Ps contribution to the total cross section 16 and an Sd-Ss interference of a few percent [17], measuring unpolarized angular distributions.

We conclude that it should be of high priority to proceed with the study of the main inelastic channel in $N N$ interactions, both experimentally by means of high statistics experiments that allow the extraction of angular distributions of spin-dependent observables, and theoretically by extending the many existing models concerning the Ss contribution to the total cross section to also include higher partial waves. 


\section{Acknowledgements}

We thank Dr. C. Hanhart for making the theoretical predictions of the model of the Jülich group available and one of the authors, P.T.E., thanks Dr. C. Hanhart for useful discussions and comments. This work has been supported by the US National Science Foundation under Grants PHY95-14566, PHY96-02872, PHY97-22556, and by the US Department of energy under Grant DOE-FG02-88ER40438.

\section{REFERENCES}

1. H.O. Meyer et al., submitted to Phys. Rev. Lett., nucl-ex/9907017

2. H.O. Meyer, Proc. $8^{\text {th }}$ Int. Conf. on the Structure of Baryons, Bonn, 22-26 Sep (1998)

3. H.O. Meyer et al., Phys. Rev. Lett. 81 (1998) 3096

4. Swapan K. Saha, W.W. Daehnick et al., Phys. Lett, in print, nucl-ex/9907016

5. P. Thörngren Engblom et al., Proc. Int. Conf. Mesons and Light Nuclei, PraguePruhonice, Aug 31-Sep 4 1998, World Scientific, in print, nucl-ex/9810013

6. T. Rinckel et al., submitted to Nucl. Inst. Meth.

7. T. Wise, A.D. Roberts and W. Haeberli, Nucl. Inst. Meth. A336 (1993) 410

8. H.O. Meyer et al., Nucl. Phys. A 539 (1992) 633

9. R.C. Hanna, Proc. 2nd Int. Symp. Polarization Phenomena, eds. P. Huber and H. Schopper, Birkhaeuser, Basel (1966) 280

10. W. Haeberli et al., Phys. Rev. C55 (1997) 597

11. F. Rathmann et al., Phys. Rev. C58 (1998) 658

12. B. von Przewoski et al., Phys. Rev. C58 (1998) 1897

13. B. Lorentz et al., submitted to Phys. Rev.

14. C. Hanhart et al., Phys. Lett. B 444 (1998) 25,

15. C. Hanhart et al., contribution to these proceedings

16. J. Zlomańczuk et al., contribution to these proceedings

17. J. Zlomańczuk et al., Phys. Lett. B436 (1998) 251, 